\title{
Nouvelles dispositions pour les médecins à partir de juillet 2021
}

\section{Bruno Henggi}

Responsable Public affairs

Après presque vingt ans, la gestion provisoire de l'admission des médecins dans le secteur ambulatoire est remplacée par une nouvelle base légale durable. Adoptée par les Chambres fédérales lors de la session d'été, cette modification de loi devrait entrer en vigueur le $1^{\text {er }}$ juillet 2021. La FMH a suivi de près les débats parlementaires et a réussi à faire introduire dans la loi des critères de qualité pour l'admission des médecins.

Pour qu'un médecin soit admis à facturer son activité ambulatoire à la charge de l'assurance obligatoire en Suisse, il devra désormais avoir exercé au moins pendant trois ans dans un établissement suisse de formation reconnu dans la discipline demandée pour l'admission. Ce qui est nouveau c'est que ces trois ans doivent être accomplis dans la discipline demandée.

\section{Exercer dans la discipline demandée}

Si une spécialiste en cardiologie souhaite être admise à pratiquer en tant que cardiologue, elle n'a plus le choix entre 4400 établissements de formation pour accomplir les trois ans mais seulement entre 60 (état: 11 juin 2020). Le nombre restreint de postes spécialisés disponibles limitera à lui seul les admissions. En revanche, un jeune médecin qui vise une admission en médecine interne générale aura le choix (le plus large) entre 1675 établissements de formation.

Les cantons peuvent par ailleurs influer sur l'admission des fournisseurs de prestations du secteur ambu-

\begin{tabular}{ll}
\hline Discipline & Nombre d'EFP* \\
\hline Médecine interne générale & 1675 \\
\hline Chirurgie de la main & 25 \\
\hline Cardiologie & 60 \\
\hline Psychiatrie et psychothérapie & 417 \\
\hline Radiologie & 443 \\
\hline Chirurgie orthopédique & 73 \\
\hline Total & $\mathbf{4 4 9 8}$ \\
\hline * EFP = établissement de formation postgraduée. \\
$\begin{array}{l}\text { (Source: registre des établissements certifiés, } \\
\text { https://www.registre-isfm.ch, 11 juin 2020) }\end{array}$
\end{tabular}

latoire via les listes hospitalières et les mandats de prestations.

\section{Attester les compétences linguistiques}

Pour être admis à facturer à la charge de l'assurance obligatoire, les médecins devront fournir la preuve qu'ils disposent des compétences linguistiques nécessaires dans la région dans laquelle ils exercent. Pour ce faire, ils passeront un test de langue en Suisse.

\section{Exception}

Les médecins sont libérés de cette obligation lorsqu'ils sont titulaires d'une maturité gymnasiale suisse dont l'une des disciplines fondamentales est la langue officielle de la région dans laquelle ils exercent. Conformément à l'Ordonnance sur la reconnaissance des certificats de maturité gymnasiale (ORM), les disciplines fondamentales sont, entre autres, la langue première, une deuxième et une troisième langue nationale. En tant que rapporteur de la commission devant le Conseil des Etats, le conseiller aux Etats Pirmin Bischof a interprété la réglementation comme suit le 4 décembre 2019: «Votre commission était majoritairement de l'avis, [...] qu'un médecin zurichois qui veut exercer à Genève ne doit pas passer de test de langue.» La conseillère nationale Ruth Humbel a donné, au nom de la commission, l'explication suivante au Conseil national le 3 mars 2020: "Concrètement, cela signifie par exemple qu'un médecin qui a obtenu une maturité suisse alémanique avec le français comme discipline d'examen ne doit pas passer de test de langue pour être admis à exercer en 
tant que médecin en Suisse romande, tout comme un Tessinois titulaire d'une maturité tessinoise, dont une discipline d'examen est l'allemand, ne doit pas passer de test de langue pour la Suisse alémanique.» Le conseiller fédéral en charge du dossier s'est également rallié à cette interprétation le 3 mars 2020. "Je me dois de préciser que nous sommes d'accord avec l'interprétation qui semble prévaloir, si j'ai bien compris, dans la commission, à savoir que lorsqu'un médecin a suivi une formation avec une maturité en Suisse, s'il a obtenu une maturité en allemand mais qu'il a passé un examen avec le français comme deuxième langue nationale, alors il doit aussi pouvoir s'installer dans la partie francophone du pays sans devoir faire un examen supplémentaire. Si, par contre, nous avons affaire par exemple à un étudiant francophone à Lausanne qui a passé ses examens avec comme deuxième langue l'allemand et pas l'italien, alors il pourra s'installer à Zurich sans examen de langue mais pas à Lugano. Si ce dernier veut s'installer à Lugano, il devra encore montrer qu'il a les compétences linguistiques nécessaires pour y pratiquer.»

\section{Des nombres maximaux de médecins, mais...}

Les cantons définissent les nombres maximaux de médecins dans une ou plusieurs spécialités et tiennent compte, pour cela, de l'évolution générale du taux d'activité des médecins. Les cantons peuvent intervenir de manière différenciée en fonction de la région et consultent préalablement les fédérations des fournisseurs de prestations, des assureurs et des patients. Avec la nouvelle réglementation, les nombres maximaux s'appliquent à tous les médecins souhaitant exercer dans le secteur ambulatoire, à savoir dans un cabinet médical, dans une institution de soins ambula- toires dispensés par des médecins ou dans le secteur ambulatoire des hôpitaux, alors que ce n'était pas le cas avec l'ancien régime des admissions.

\section{...pas de droit de recours...}

Aucun droit de recourir contre les décisions cantonales concernant le calcul et les nombres maximaux de médecins fixés n'est accordé aux assureurs. Cela relativise l'importance de l'obligation faite aux cantons de fixer les nombres maximaux de médecins.

\section{...et pas d'obligation}

Les cantons ont désormais la possibilité - sans obligation - de stopper toute admission supplémentaire dans une discipline déterminée, et ce indépendamment des nombres maximaux de médecins fixés. Un canton peut prévoir qu'aucune nouvelle admission à pratiquer à la charge de l'assurance obligatoire des soins (AOS) n'est délivrée dans une spécialité lorsque les coûts annuels par assuré dans cette spécialité augmentent davantage que les coûts annuels des autres spécialités (dans ce canton) ou augmentent davantage que la moyenne suisse des coûts annuels de cette spécialité.

\section{Surveillance des caisses maladie}

Les cantons décident si un médecin remplit les critères d'admission. Ils se chargent donc désormais de l'admission administrative de police sanitaire et de l'admission à facturer à la charge de l'AOS. Si les fournisseurs de prestations sont admis à pratiquer par un canton et qu'ils exercent à la charge des assureurs maladie, c'est aux assureurs qu'il appartient de vérifier l'économicité, l’efficacité et la qualité.

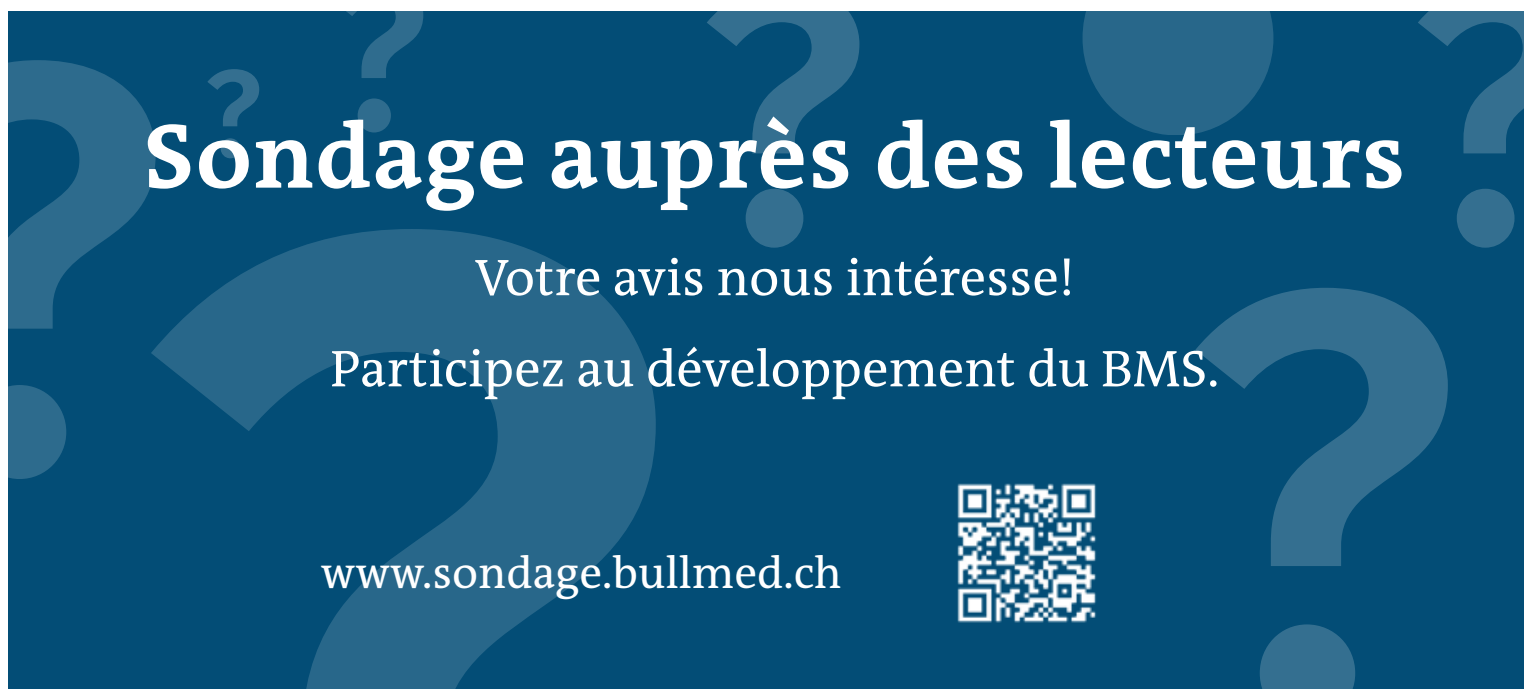

